\title{
Preimpresiones en biomedicina: ¿alternativa o complemento al modelo tradicional de publicación?
}

\author{
Guillermo Aquino-Jarquin ${ }^{1}$, Josefina de Monserrat Valencia-Reyes ${ }^{1}$, Abraham Silva-Carmona ${ }^{1,2}$ y \\ Javier Tadeo Granados-Riverón ${ }^{1}$ \\ ${ }^{1}$ Laboratorio de Investigación en Genómica, Genética y Bioinformática, Hospital Infantil de México Federico Gómez; '2Servicio de Patología, Hospital \\ General de México. Ciudad de México, México
}

\section{Resumen}

La revisión por pares es un sistema que ha permitido el control de calidad de los manuscritos enviados para publicación en revistas científicas durante más de tres siglos. Sin embargo, debido a su relativa lentitud y otras desventajas, algunos investigadores (principalmente en las áreas de la física y las matemáticas) iniciaron hace algunas décadas la difusión electrónica de manuscritos aún no sometidos a una revista de publicación formal. La popularización de esta práctica condujo al establecimiento de repositorios permanentes como ArXiv, a los que es posible enviar preimpresiones de forma gratuita y que a la vez permiten la búsqueda y descarga de los trabajos que contienen sin cargo para el lector. En las ciencias biomédicas la adopción de este sistema ha sido más lenta que en las ciencias exactas e intentos previos como e-biomed, Netprints y Nature Precedings no prosperaron. Una nueva generación de repositorios como bioRXiv, inspirado en ArXiv, parece gozar de una creciente aceptación entre investigadores biomédicos. Aquí discutimos el potencial papel de este sistema emergente para establecer la primicia de descubrimientos en biomedicina y el mejoramiento de manuscritos antes de su sometimiento a revistas científicas, así como para otras aplicaciones que podrían implementarse en la medida en que el modelo se popularice.

PALABRAS CLAVE: Preimpresiones. Revisión por pares. Repositorios. Publicación científica Publicaciones biomédicas.

\begin{abstract}
The peer-review system has allowed the quality control of the manuscripts submitted for publication to scientific journals for over three centuries. However, due to its relative slowness and other drawbacks, some researchers, mainly in the areas of Physics and Mathematics, started some decades ago to propagate, by electronic means, manuscripts not yet submitted to a journal for formal publication. The dissemination of this practice led to the establishment of permanent repositories like ArXiv, to which preprints can be sent to be published whitou charge, allowing also the search and download of the works they contain with no payment required from the reader. In biomedical sciences, the adoption of the system has been slower than in the exact sciences and previous attempts like e-biomed, Netprints, and Nature Precedings did not prosper. A new generation of repositories like bioRXiv, inspired by ArXiv, seems to enjoy an increasing acceptance among biomedical researchers. Here, we discuss the potential role of this emerging system to establish discovery priority in biomedicine and to improve manuscripts before they are submitted to scientific journals besides other applications which could be implemented in the extent that the model becomes more popular.
\end{abstract}

KEY WORDS: Preprints. Peer-review. Repositories. Scientific publication. Biomedical publishing.

\author{
Correspondencia: \\ Javier Tadeo Granados-Riverón \\ Dr. Márquez, 162 \\ Col. Doctores \\ C.P. 06720, Ciudad de México, México \\ E-mail: JavierTGranados@ himfg.edu.mx
}

Fecha de recepción en versión modificada: 18-07-2016

Fecha de aceptación: 27-07-2016

DOI://dx.doi.org/10.24875/GMM.17002770
Gac Med Mex. 2018;154:87-91

Contents available at PubMed www.gacetamedicademexico.com 


\section{Introducción}

Desde sus inicios, cuando el naturalista Henry OIdenburg lo implementó en Philosophical Transactions of the Royal Society en la segunda mitad del siglo XVII, el sistema de revisión por pares (o peer-review, en inglés) se ha constituido como el mecanismo más usado para fomentar la calidad de la literatura científica en todo el mundo. Salvo un breve periodo que incluyó el uso del fax, hasta hace pocas décadas, la revisión por pares involucró el intercambio físico de textos impresos e ilustraciones ${ }^{1}$. Igualmente, ya desde finales del siglo pasado, la efectividad y consistencia del modelo tradicional de revisión por pares han sido cuestionadas, como lo ha sido la utilidad de las medidas propuestas desde entonces para mejorar el proceso².

En un proceso tradicional de publicación, el editor de la revista que recibe un manuscrito decide, basado en su tema, calidad, originalidad y rigor, además del interés potencial de sus lectores en el mismo, si este debe rechazarse o bien enviarse a revisión por pares, que usualmente llevan a cabo de dos a tres expertos en el tema. Tras su análisis, cada revisor emite una recomendación al editor en el sentido de si el manuscrito debe aceptarse para publicación, rechazarse o bien considerarse para un nuevo análisis, una vez que los autores respondan adecuadamente a los cuestionamientos vertidos en los reportes de los revisores, y que pueden incluir la petición de cambios en la redacción del trabajo, análisis de los datos 0 , con frecuencia, la realización de nuevos experimentos. Eventualmente, tras un número variable de rondas de revisión por pares, el manuscrito es finalmente aceptado o rechazado para publicación. Todo este proceso puede comprender desde algunas semanas hasta más de un año $\mathrm{y}$, en caso de que el manuscrito sea rechazado y sometido a una nueva revista, substancialmente más tiempo.

A pesar de que el uso de Internet ha agilizado enormemente el intercambio de la información utilizada para la revisión por pares, evidencia reciente revela que el promedio de tiempo necesario entre recepción y publicación de un manuscrito en Nature se ha incrementado de 85 a poco más de 150 días en la última década, mientras que para PLoS ONE el mismo parámetro se ha elevado de 37 a 125 días en aproximadamente el mismo periodo ${ }^{3}$. Al menos en parte, esto puede ser atribuido al incremento en la cantidad de datos que son necesarios para lograr la publicación de un trabajo. Una comparación entre artículos publicados en Cell, Nature y The Journal of Cell Biology reveló que la cantidad de paneles en figuras entre 1984 y 2014 se ha incrementado de dos a cuatro veces ${ }^{4}$.

En las últimas décadas, particularmente en biomedicina, tanto el aumento explosivo en el número de revistas científicas como el consecuente incremento en el número de artículos que se publican anualmente no han sido acompañados por un aumento proporcional en el número de potenciales revisores. Adicionalmente, el hacer una revisión de calidad de un artículo requiere una inversión de tiempo importante, que, de manera global, no reditúa en un beneficio directo proporcional a la misma. En el caso de México en particular, el fungir como revisor no es reconocido como un criterio que facilite el ingreso o permanencia a esquemas como el Sistema Nacional de Investigadores.

Este panorama de alto volumen y poco incentivo, sin duda, ha contribuido a la disminución del rigor y la calidad de los procesos de revisión por pares, particularmente en publicaciones con factor de impacto intermedio y bajo. Se ha estimado, por ejemplo, que el fraude científico se incrementó aproximadamente 10 veces entre 1975 y $2012^{5}$.

\section{Las preimpresiones o preprints}

En respuesta a los problemas inherentes a la revisión por pares tradicional, algunos investigadores, principalmente en física, optaron por distribuir sus descubrimientos, observaciones y modelos directamente a potenciales lectores en el formato digital TeX, antes o en lugar de someterlos a una revista para su revisión por pares y publicación. Estas preimpresiones (preprints o e-prints, en inglés) fueron primeramente distribuidas a través de correo electrónico. A inicios de los años noventa se creó un repositorio de preimpresiones, que ahora es conocido como ArXiv y es mantenido por la Universidad de Cornell. ArXiv está actualmente disponible en Internet y ha tenido un inmenso éxito, conteniendo a la fecha más de un millón de preimpresiones en los campos de física, matemáticas, ciencias de la computación, estadística, finanzas y biología cuantitativa, convirtiéndose en el principal medio de difusión en esas disciplinas ${ }^{6}$.

En las ciencias biomédicas la propuesta inicial de aplicar el modelo de la preimpresión fue llamada e-biomed, promovida por los Institutos Nacionales de Salud en EE.UU. en 19997. A pesar de que la idea no 
prosperó, dio origen a PubMed Central, un repositorio que hasta la fecha hace disponibles gratuitamente un gran número de artículos publicados en el sistema tradicional.

Más tarde, tanto BMJ (Netprints) $)^{8}$ como Nature (Nature Precedings $)^{9}$ iniciaron sendos servidores de preimpresiones con énfasis biomédico. A la fecha ya inactivos, ambos recibieron miles de trabajos durante varios años a inicios del siglo, mismos que hasta hoy siguen disponibles para su descarga. En 2012 apareció F1000research, una plataforma de publicación con el concepto de revisión por pares abierta (open peer-review), donde la identidad de los revisores, así como el contenido de todos los textos originados durante el proceso son publicados y son descargables desde la plataforma junto con la versión original y todas las versiones subsecuentes del trabajo. A pesar de que F1000research sí aplica una composición tipográfica y edición a los manuscritos que recibe, esta plataforma puede considerarse también como un repositorio de preimpresiones biomédicas, dado que el texto inicial es publicado antes de ser sometido a este proceso de la revisión por pares abierta ${ }^{10}$. Otro repositorio, PeerJ Preprints ${ }^{11}$, inició operaciones publicando sus primeras preimpresiones en abril de 2013, contabilizando a la fecha varios miles de trabajos en biomedicina.

A finales de 2013, como respuesta al creciente interés de los investigadores biomédicos en $\mathrm{ArXiv}^{12}$, Cold Spring Harbor Laboratory lanzó un servidor para preimpresiones para las áreas biomédicas llamado bioRXiv ${ }^{13}$. La plataforma bioRXiv cobró notoriedad cuando la ganadora del premio Nobel, Carol Greider, de la Universidad Johns Hopkins, cargó una prepublicación en ella donde propuso un nuevo modelo para la regulación de la longitud de los telómeros ${ }^{14}$. Otros casos sonados incluyeron prepublicaciones por grupos en el Cold Spring Harbor Laboratory, como la del neurocientífico Steve Shea, cuyo manuscrito había permanecido para entonces más de año y medio en revisión por pares ${ }^{15}$.

Una vez que un trabajo es enviado a bioRXiv y antes de que sea publicado en Internet, en cuestión de horas se completa un proceso básico de filtrado por contenido plagiado, ofensivo, no científico o pseudocientífico, pero que no incluye edición o composición tipográfica. Así mismo, las políticas del repositorio establecen que un trabajo puede ser enviado antes 0 durante un proceso de revisión por pares tradicional en una revista, pero no después de que este ha sido publicado. En el momento en que el texto es
Tabla 1. Repositorios de preimpresiones mencionadas y sus direcciones en la web

\begin{tabular}{ll}
\hline Repositorio & Dirección en la web \\
\hline ArXiv & http://arxiv.org/ \\
bioRXiv & http://biorxiv.org/ \\
F1000Research & http://f1000research.com/ \\
Nature Proceedings & http://precedings.nature.com/ \\
Netprints & http://staticweb.bmj.com/clinmed/ \\
PeerJ Preprints & http://peerj.com/preprints/ \\
\hline
\end{tabular}

publicado en Internet se le asigna un identificador de objeto digital (o DOI, por sus siglas en inglés) y versiones revisadas del mismo pueden ser enviadas por los autores en cualquier momento, pero, dado que son citables desde su publicación en el repositorio, los manuscritos no pueden ser retirados ${ }^{13}$. La entrada correspondiente a cada preimpresión en bioRXiv incluye el texto del resumen, hipervínculos para descargar la versión actual y las versiones anteriores de la preimpresión en formato PDF, así como una interface que permite citarla, compartirla mediante múltiples redes sociales o mediante correo electrónico, así como dejar comentarios previo inicio de sesión en Discus, Facebook, Twitter o Google. Adicionalmente se promueve el contacto directo con los autores mediante correo electrónico. Así mismo, las preimpresiones poseen una leyenda prominente que consigna que los trabajos no han sido aprobados en un proceso de revisión por pares y el repositorio advierte que los artículos que contiene no han sido aún aceptados o avalados en manera alguna por la comunidad científica o médica. En la tabla 1 se consignan las direcciones en la web de los repositorios de preimpresiones referidos en el texto.

\section{Ventajas y desventajas del modelo}

El modelo de publicación actual permite que, con cierta frecuencia, la primicia en un descubrimiento pueda otorgarse en función de la diferente velocidad de la revisión por pares en diferentes revistas en el caso de manuscritos reportando un mismo descubrimiento, de calidad y complejidad comparables y sometidos dentro de un intervalo corto de tiempo. Por ello, se ha planteado que el uso de preimpresiones contribuiría a un reconocimiento más justo en los descubrimientos biomédicos, si este se acompaña de una revisión por pares rigurosa simultánea o posteriormente ${ }^{16}$. 
Sin embargo, este uso de las preimpresiones puede ser un arma de doble filo. Por un lado, permite establecer el día de liberación en el repositorio como el punto en el tiempo en el que el descubrimiento se anuncia a la comunidad científica. En las revistas tradicionales usualmente se consigna la fecha de primera recepción del manuscrito, sin embargo, esta solo se hace pública si el artículo es aceptado y cuando ha sido aceptado. Por otro lado, en áreas de la biomedicina que son altamente competitivas, una potencial desventaja de hacer público el descubrimiento en una preimpresión consistiría en alertar a grupos competidores respecto al progreso de una investigación en particular y con ello acelerar su decisión de someterlo a una revisión por pares tradicional. En la medida en que la cultura de las preimpresiones se extienda en biomedicina, así como ocurre ya en muchas ciencias exactas, los revisores podrían estar más dispuestos a verificar la originalidad de una idea o un descubrimiento usando tanto publicaciones en revistas como repositorios de preimpresiones. En la misma medida, el reclamar la primicia de un hallazgo que ha sido reportado previamente en una preimpresión de otro grupo o autor, sin citarla, podría volverse menos socialmente aceptable.

En contraste con la mayoría de las revistas biomédicas, en el caso de bioRXiv y otros repositorios de preimpresiones anteriores, la publicación de un trabajo es gratuita para los autores. Así mismo, el acceso a ellos, su descarga y lectura son libres y gratuitos para los usuarios finales. Esto permite que los avances reportados sean mucho más visibles, citables y utilizables inmediatamente por otros investigadores, potencialmente contribuyendo a un avance más rápido del campo en su conjunto.

La evaluación a la que se someten los trabajos enviados a los repositorios antes de que sean puestos a disposición de los lectores, por ejemplo en bioRXiv, no sustituye al proceso de revisión por pares, por lo que la calidad de los trabajos ahí contenidos es más variable en comparación con revistas arbitradas. Esto implica que la responsabilidad de evaluar la validez, originalidad e importancia de cada trabajo es transferida de los revisores a los lectores. Esto cobra especial relevancia para la utilización de las preimpresiones para medir el desempeño de los investigadores, ya que se requeriría que los dictaminadores ejercieran criterios más analíticos para diferenciar preimpresiones de distintos grados de calidad y rigor científico.

Como se describió para el caso de bioRXiv, las interfaces web de los repositorios de preimpresiones biomédicos facilitan que los usuarios del sitio comenten sobre cada trabajo. Esto tiene la enorme ventaja de que cualquiera puede aportar críticas y sugerencias a los autores, lo cual permitiría mejorar un manuscrito antes de someterlo a revisión por pares formal, proceso en el que el contenido de cada trabajo es evaluado por muy pocas personas.

Actualmente, ciertas revistas científicas imponen límites al número de palabras, figuras y referencias a los artículos que se les envían, mientras que otras no admiten la inclusión de información suplementaria. Debido a que en bioRXiv no se establecen estas restricciones, las preimpresiones podrían convertirse en valiosas fuentes de descripciones metodológicas detalladas, lo cual incrementaría las posibilidades de que los experimentos pudieran ser reproducidos exitosamente.

El empleo de preimpresiones también puede contribuir a la disminución del sesgo de publicación, en el sentido en que las revistas tradicionales tienen más probabilidades de contener resultados positivos. Bajo esta premisa, los investigadores pueden estar más inclinados a publicar sus resultados negativos, una valiosa fuente de información, en repositorios de preimpresiones.

Una dificultad para que la práctica de liberar preimpresiones sea más extendida es que, al momento, algunas revistas biomédicas, por ejemplo las que pertenecen al Cell Publishing Group, no aceptan artículos que hayan sido previamente prepublicados en plataformas como bioRXiv. Una base de datos en línea, alojada en Wikipedia, mantiene un registro actualizado sobre las políticas de las casas editoriales respecto a la aceptación de artículos que hayan sido objeto de una preimpresón (https://en.wikipedia.org/ wiki/List_of_academic_journals_by_preprint_policy).

\section{La Reunión ASAPbio}

Debido a la prominencia que las preimpresiones han ido cobrando en biomedicina, en parte gracias a la difusión de bioRXiv, alrededor de 70 destacados investigadores, representantes de la industria, agencias de financiamiento científico, así como representantes de casas editoriales, todos ellos interesados en este sistema emergente, se reunieron a mediados de febrero de 2016 durante la Reunión ASAPbio (del inglés Acelerating Science and Publication in biology $)^{6}$, para discutir cómo los diferentes tipos de actores pueden propiciar un uso racional y productivo de los repositorios y los textos ahí almacenados. En el análisis de 
las conclusiones de los académicos se destaca que, con el fin de fomentar la producción de preimpresiones por parte de los investigadores biomédicos, es necesario que existan formas sistemáticas, como motores de búsqueda, para asociar a un artículo publicado en una revista a la o las preimpresiones que le antecedieron. Para los representantes de las agencias de financiamiento, las preimpresiones pueden ser valiosas para tener una visión más actualizada de la productividad de un investigador y una visión más oportuna de las reacciones de la comunidad científica a sus contribuciones. Los representantes de las casas editoriales concluyeron que las preimpresiones y las revistas tradicionales deben jugar papeles diferentes pero complementarios en la difusión de los avances científicos. También puntualizaron que los comentarios a una preimpresión podrían servir de guía para facilitar la labor de los revisores pares y que sería aconsejable la inclusión en los repositorios de mecanismos para el sometimiento directo de las preimpresiones a las revistas tradicionales. En votaciones privadas y opcionales durante la reunión, la gran mayoría de los asistentes optó por favorecer las opiniones de que las preimpresiones pueden usarse para establecer la primicia de un hallazgo y de que pueden también usarse como evidencia en evaluaciones de la productividad de los investigadores.

\section{Conclusiones y consideraciones finales}

El someter un manuscrito a un servidor de preimpresiones puede ser muy útil para mejorar su calidad, gracias a los comentarios que reciba, antes de someterlo a una revista para revisión por pares formal. Adicionalmente, las preimpresiones pueden emplearse para establecer la primicia de un descubrimiento siempre y cuando, a juicio de investigador, las conclusiones a las que se llega en el trabajo estén justificadas por la metodología y los resultados plasmados en él. Ambos aspectos deben considerarse con cautela en líneas de investigación altamente competitivas, donde el riesgo de perder prioridad por márgenes pequeños es alto.

En la medida en que las preimpresiones se afiancen como un medio aceptable y visible de propagación del conocimiento científico en biomedicina, este instrumento será cada vez más útil para que estas publicaciones se vuelvan un criterio para el otorgamiento de cátedras, estímulos y financiamiento a los investigadores, un cambio que, sin embargo, debe estar acompañado por la adquisición de un enfoque crítico por parte de los dictaminadores involucrados para discriminar aquellas preimpresiones que son robustas científicamente de las que no lo son.

A pesar de que la premisa de que en biomedicina el sistema actual de publicación en revistas está descompuesto más allá de reparación ${ }^{17}$ es sin duda catastrofista, es innegable que este proceso está evolucionando de forma mucho más rápida en los últimos años gracias a su conjunción con los inmensos avances en tecnologías de la información y telecomunicaciones. El uso de las preimpresiones es solo una de las innovaciones propiciadas de esta forma y gracias a otras tipos de plataformas como los repositorios de datos para ciencia abierta y de plataformas de revisión por pares pospublicación, entre otras tantas, es posible que en el futuro observemos el desarrollo de un ecosistema entero para la propagación de la información generada por la investigación biomédica. Como apuntaba Francis Crick, físico, neurocientífico y codescubridor de la estructura del ADN, «En la naturaleza las especies híbridas son usualmente estériles, pero en ciencia, lo opuesto es frecuentemente verdadero ${ }^{18}$.

\section{Bibliografía}

1. Spier R. The history of the peer-review process. TRENDS Biotechnol. 2002;20:357-8.

2. Goldbeck-Wood S. Evidence on peer review - scientific quality control or smokescreen? BMJ. 1999;318:44-5

3. Powell K. Does it take too long to publish research? Nature. 2016;530:148-51.

4. Vale RD. Accelerating scientific publication in biology. Proc Natl Acad Sci U S A. 2015;112:13439-46.

5. Fang FC, Steen RG, Casadevall A. Misconduct accounts for the majority of retracted scientific publications. Proc Natl Acad Sci. 2012;109:17028-33.

6. Berg JM. Preprints for the Life Sciences. Science. 2016;352:899-901.

7. Homan JM. E-biomed. Bull Med Libr Assoc. 1999;87:485-6.

8. Delamothe T, Smith R, Keller MA. Netprints: the next phase in the evolution of biomedical publishing. BMJ. 1999;319:1515-6.

9. Free market science. Nat Cell Biol. 2007:9:721.

10. Ford E. Open peer review at four STEM journals: an observational overview. Version 2. F1000Res. 2015;4:6.

11. Callaway E. Biomedical journal and publisher hope to bring preprints to life. Nat Med. 2013;19:512

12. Callaway E. Geneticists eye the potential of arXiv. Nature. 2012;488:19.

13. Callaway E. Preprints come to life. Nature. 2013;503:180.

14. Greider CW. Regulating telomere length from the inside out: The replication fork model. Genes Dev. 2016;30(13):1483-91.

15. Krishnan K, Lau B, Ewall G, et al. MECP2 regulates cortical plasticity underlying a learned behavior in adult female mice. Nat Commun. 2017;8:14077

16. Vale RD, Hyman AA. Priority of discovery in the life sciences. Elife. $2016 ; 5$.

17. Chalmers I, Glasziou P. Should there be greater use of preprint servers for publishing reports of biomedical science? F1000Research. 2016:5:272.

18. Crick F. What mad pursuit: a personal view of scientific discovery. Basic Books; 2008. 